\title{
Preliminary Study on The SYBR Green I RT- qPCR for Rabbit Hemorrhagic Disease Virus 2 (RHDV2) Detection
}

\author{
Zexiao YANG* \\ College of Veterinary Medicine \\ Sichuan Agricultural University \\ Chengdu, China \\ e-mail: yzxyang2003@126.com
}

Zhengqun MENG

College of Veterinary Medicine

Sichuan Agricultural University

Chengdu, China

e-mail: yzxyang2003@126.com

Xueping YAO

College of Veterinary Medicine

Sichuan Agricultural University

Chengdu, China

e-mail: yzxyang2003@126.com

\section{Yi GENG}

College of Veterinary Medicine

Sichuan Agricultural University

Chengdu, China

e-mail: yzxyang2003@126.com

\author{
Yadong LIU \\ College of Veterinary Medicine \\ Sichuan Agricultural University \\ Chengdu, China \\ e-mail: yzxyang2003@126.com
}

\section{Bo WANG}

College of Veterinary Medicine

Sichuan Agricultural University

Chengdu, China

e-mail: yzxyang2003@126.com

\section{Yin WANG}

College of Veterinary Medicine

Sichuan Agricultural University

Chengdu, China

e-mail: yaanwangyin@tom.com

Yan LI

College of Animal Science and Technology Sichuan Agricultural University

Chengdu, China

e-mail: liyan@sicau.edu.cn

\begin{abstract}
To develop a rapid method for the detection RHDV2, 4 specific primers and 12 overlapping PCR primers were designed to amplify the conserved RHDV2 specific DNA fragment according to the genome sequences of RHDV and RHDV2 published in GenBank. Based on the synthesis of a conserved part of the RHDV2 sequence using overlap extension PCR, a SYBR Green I RT-qPCR assay was first preliminary developed and evaluated in China after a series of tests, including, reaction conditions optimization, establishment of standard curve, the sensitivity and specificity tests, intra-assay, and the application tests of 30 samples. The results showed that a 435bp specific DNA fragment of the RHDV2 capsid protein(VP60)gene was synthesized in vitro and the recombinant plasmid pMD-19T-RHDV2 was constructed, and the RT-qPCR method for RHDV2 rapid detection had good specificity, sensitivity and repeatability, which performance was linear ranging from 128 copies $/ \mu \mathrm{L}$ to $1.28 \times 108$ copies $/ \mu \mathrm{L}$ for the cloned viral genomic fragments of RHDV2.The sensitivity of the RT-qPCR could reach about 128 copies $/ \mu \mathrm{L}$ of target fragments, and there was no amplification for RHDV, pGM-T-EBHSV, Pasteurella multocida, E.coli and Salmonella from rabbits detection by this method. And the application tests results showed that there were not RHDV2 in the experimentally infected samples and clinical samples.
\end{abstract}

Keywords-rabbit hemorrhagic disease; RHDV2; RT-qPCR; overlapping PCR; detection

\section{INTRODUCTION}

Rabbit hemorrhagic disease (RHD), caused by rabbit hemorrhagic disease virus (RHDV), is a highly contagious and rapidly fatal viral disease of both domestic and wild rabbits (Oryctolagus cuniculus) [1]. The disease is characterized with the typical pathological lesions including necrotic hepatitis, hemorrhaging and edema of lungs and trachea, short incubation period (24-48 h after infection) ,and high mortality rate of $60-100 \%$ to the adult rabbits[2]. Since RHD was first described in China in 1984, it has been reported in many countries successively throughout the world [3].RHD has resulted in the deaths of nearly a quarter billion wild and domestic rabbits, and it was responsible for significant losses in the rabbit industry and became an important disease in the OIE List[4]. Although the efficient commercial vaccines protect against RHDV has enabled its control in rabbitries, RHD is still endemic in some areas[4,5].In the summer 2010,a new type rabbit hemorrhagic disease(named RHD2) caused by Rabbit 
hemorrhagic disease virus type 2(RHDV2 or RHDVb)was reported in France both in rabbitries and in wild populations, which generates the similar pathogenic changes with RHD with about 20\% mortality[6].RHD2 can affect the rabbit kits under 30 days of age, RHD-vaccinated rabbits and other lagomorphs like different hare species[7].Since the first emergence report of RHD2 in France,RHD2 has spread to several European countries and the African continent, such as Italy, Spain, Germany, Portugal mainland, England, Wales, several islands of the Azores, etc[8]. There are no the appearances reports about RHD2 cases in China reported until now, but as the development of international economic and trade such as rabbits and its related products introduction, it is very important and necessary to carry out the researches about the methods for RHDV2 detection to strengthen the inspection and quarantine. In this study a SYBR Green I RT-qPCR (real-time RT-PCR) method for RHD2 diagnosis was preliminary established and evaluated based on the target gene fragment synthesis in vitro of RHDV2 VP60 by overlapping PCR.

\section{MATERIALS AND METHODS}

A. Reagents and Samples.

DNA Maker DL2000, Primerscript RT reagent Kit (BK1901), RNAiso Plus and pMD19-T Vector kit were provided by the TaKaRa Biotechnology (Dalian) Co.,Ltd. DH5 $\alpha$ competent cells, 2×Taq PCR MasterMix (KT201), $2 \times$ Sybr qPCR Mix (261932AX), Pfu DNA polymerase, TIANprep Mini Plasmid Kit (DP103), and TIANgel Midi Purification Kit (DP209) were provided by the TIANgen Biotech(Beijing) Co., Ltd, RHDV-Sch01, Vp60 gene fragments of European brown hare syndrome virus (pGM-T- EBHSV), Pasteurella multocida, E.coli and Salmonella from rabbits were provided by Sichuan Agricultural University animal quarantine Lab, 5 RHDV experimentally samples infected with the RHDV-Sch01 and 25 clinical samples were collected and stored at $-70{ }^{\circ} \mathrm{C}$.

\section{B. Primer Preparation}

According to the VP60 gene sequences of RHDV and RHDV2 published in GenBank, 2 primers (P1/P2)for RHDV RT-PCR, 12 overlapping PCR oligo primers and 2 specific primers for RHDV2 real-timeRT-PCR (listed in table I) were carefully designed using DNAStar and Beacon designer 8 subsequently. All of these primers were prepared by the BGI Sequencing Co., Ltd.

TABLE I. PRIMERS USED FOR RHDV2 SYBR GREEN I RT-QPCR STUDY

\begin{tabular}{|c|c|c|c|}
\hline $\begin{array}{c}\text { Task and } \\
\text { Primer name }\end{array}$ & Sequence $\left(5^{\prime}-3^{\prime}\right)$ & $\begin{array}{l}\text { Products } \\
\text { Size(bp) }\end{array}$ & $\begin{array}{r}\text { Reference } \\
\text { sequence }\end{array}$ \\
\hline RHDV RT-PCR & & & \\
\hline P1 & GGGTGTCATATCCACСССАAA (1269-1289) & 441 & DQ205345 \\
\hline P2 & CCCAGGTTGAACACGAG (1709-1693) & & \\
\hline \multicolumn{4}{|l|}{$\begin{array}{l}\text { RHDV2 Overlap } \\
\text { extension PCR }\end{array}$} \\
\hline $\mathrm{F}$ & CATATCCACCССAAACAGT (1275-1293) & 435 & KP129339 \\
\hline $\mathrm{R}$ & CCCAAGTTGTACACAAGC (1709-1692) & & \\
\hline F1 & $\begin{array}{l}\text { TATCCACCCCAAACAGTAATGCCGTCACGTACACACCTCA } \\
\text { GCCAAACAGGA }\end{array}$ & & \\
\hline F2 & $\begin{array}{l}\text { GCTCCTATTGGCAAGAACACACCCATCATGTTCGCGTCTGT } \\
\text { TGTTAGGCGCACCGGCGA }\end{array}$ & & \\
\hline F3 & $\begin{array}{l}\text { GAACCCAGTACGGCGCGGGATCACAACCACTGCCGGTGA } \\
\text { CAGTTGGACTCTCACTGAAC }\end{array}$ & & \\
\hline F4 & $\begin{array}{l}\text { GTTCTTTGTTTGGCAGCTGAATTTCGCTTCCGGTTTCATGG } \\
\text { AACTTGGCTTGAGTGTTG }\end{array}$ & & \\
\hline F5 & $\begin{array}{l}\text { TCAGCCACCCTCATTGACCTGTCAGAACTTGTTGACATCCG } \\
\text { CCCTGTGGGACCCAGACC }\end{array}$ & & \\
\hline R1 & $\begin{array}{l}\text { CTTGCCAATAGGAGCGGCAGCAGGGGTGCCAGGTGCATTG } \\
\text { ACAATCCTGTTTGGCTGAG }\end{array}$ & & \\
\hline $\mathrm{R} 2$ & $\begin{array}{l}\text { CGCCGTACTGGGTTCCGTTAGCTGAACCGGCCTCAGCGTT } \\
\text { GATGTCGCCGGTGCGCCTA }\end{array}$ & & \\
\hline R3 & $\begin{array}{l}\text { TGCCAAACAAAGAACTGCCCAGGCATAAGTGCCGACGAGT } \\
\text { AATTGTTCAGTGAGAGTCC }\end{array}$ & & \\
\hline $\mathrm{R} 4$ & $\begin{array}{l}\text { AATGAGGGTGGCTGAAGCCCCCGTCCCTGCATAGAAGTAC } \\
\text { CCATCAACACTCAAGCCAA }\end{array}$ & & \\
\hline R5 & $\begin{array}{l}\text { CCCAAGTTGTACACAAGCGTGCTTGTGGACGGTCTGGGTC } \\
\text { CCACA }\end{array}$ & & \\
\hline RHDV2real-timePCR & & & \\
\hline R2P1 & AACCACTGCCGGTGACAG(1469-1486) & 125 & KP129339 \\
\hline R2P2 & ATCAACACTCAAGCCAAG(1593-1576) & & \\
\hline
\end{tabular}

\section{Synthesis of RHDV2 Target VP60 Gene Fragment}

The conserved target VP60 gene fragment of RHDV2 was synthesized in vitro using an overlap extension PCR method as described in[9],including the primary extension reactions, secondary extension reactions and PCR. 
The primary extension reaction volume $(50 \mu \mathrm{L})$ contains $5 \mu \mathrm{L} 10 \times$ Pfu DNA polymerase buffer, $6 \mu \mathrm{L}$ of dNTPs (2.5mmol/L), $1 \mu \mathrm{L}(10 \mu \mathrm{mol} / \mathrm{L})$ of each of the overlapping oligo primers (F1/R1, F2/R2, F3/R3, F4/R4 or F5/R5), $1 \mu \mathrm{L}$ of Pfu DNA polymerase, and $36 \mu \mathrm{L}$ ddH2O. And the secondary extension reaction volume $(50 \mu \mathrm{L})$ containing $1 \mu \mathrm{L}$ $10 \times$ Pfu DNA polymerase buffer, $8 \mu \mathrm{L}$ of dNTPs (2.5mmol/L), $1 \mu \mathrm{L}$ of Pfu DNA polymerase, and $20 \mu \mathrm{L}$ of each of the two overlap previous extension reaction products. The reaction conditions were $94^{\circ} \mathrm{C}$ for $30 \mathrm{~s}$ and $72^{\circ} \mathrm{C}$ for $15 \mathrm{~min}$.

The PCR reaction volume $(50 \mu \mathrm{L})$ containing $25 \mu \mathrm{L}$ of $2 \times$ Taq PCR MasterMix, $1 \mu \mathrm{L}(10 \mu \mathrm{mol} / \mathrm{L})$ of each of the primers $(\mathrm{F} / \mathrm{R}), 5 \mu \mathrm{L}$ of the last secondary extension reaction products and $18 \mu \mathrm{L}$ ddH2O. The PCR was conducted as follows: denaturing at $95^{\circ} \mathrm{C}$ for $5 \mathrm{~min}$, followed by 35 cycles at $94^{\circ} \mathrm{C}$ for $40 \mathrm{~s}, 57^{\circ} \mathrm{C}$ for $30 \mathrm{~s}$, and $72^{\circ} \mathrm{C}$ for $30 \mathrm{~s}$, then terminated by an elongation at $72^{\circ} \mathrm{C}$ for $8 \mathrm{~min}$. In all, $5 \mu \mathrm{L}$ of PCR products were analyzed using $10 \mathrm{~g} / \mathrm{L}$ agarose gel electrophoresis, then photographed under a UV imaging system (Bio-Rad).

The PCR products were subsequently separated and purified according to the TIANgel Midi Purification Kit (DP209) instructions and were then directly cloned into the pMD19-T vector followed by a transformation into the DH5 $\alpha$ competent cells. Then, the recombinant plasmids constructed were extracted using a TIANprep Mini Plasmid Kit and were subsequently identified by PCR (using F/R) and by sequencing analysis [9]. And the positive recombinant plasmid was extracted and stored at $-20^{\circ} \mathrm{C}$ for the template used in this study.

\section{Cloning of Corresponding RHDV VP60 Target Fragment}

The RNA extraction of RHDV and RT reaction were subsequently performed by using the RNAiso Plus reagent (BK1501) and PrimeScriptTM RT Reagent kit (BK1901).Using P1/P2 as primers and $2 \mu \mathrm{L}$ of the $\mathrm{RT}$ reaction products as templates, the corresponding RHDV VP60 target fragments were amplified by PCR (the PCR reaction system and procedure were both same as above), and then were separated, purified, cloned into the pMD19-T vector and identified as above.

\section{E. Optimization of RHDV2 SYBR Green I RT-qPCR Reaction Temperature}

The optimum reaction conditions for specific primers R2P1 and R2P2 was determined by a real-time PCR assay using the recombinant plasmids contained the synthesized RHDV2VP60 gene fragment as the templates. The reaction volume $(20 \mu \mathrm{L})$ containing $10 \mu \mathrm{L}$ of $2 \times$ Sybr qPCR Mix, $1 \mu \mathrm{L}$ $(5 \mu \mathrm{mol} / \mathrm{L})$ of each of the primers (R2P1/R2P2), $2 \mu \mathrm{L}$ of the recombinant plasmids and $6 \mu \mathrm{L}$ ddH2O. The conditions are same as the CFX2StepAmp+Melt but different from a gradient of annealing degree from $59^{\circ} \mathrm{C}$ to $65^{\circ} \mathrm{C}$ for $25 \mathrm{~s}$. The negative control with ddH2O was detected in parallel.

\section{F. The Standard Curve and Sensitivity Test of Qpcr}

Using the recombinant plasmids constructed above as standard control samples, which concentration was determined by a ND-1000 ultraviolet spectrophotometer (Nano Drop Co., Ltd, USA). The recombinant plasmids were diluted in a 10 -fold serial ranging from $10^{-2}$ to $10^{-10}$, and then each dilution was tested by the qPCR using the determined reaction conditions. The number of target gene fragment molecule copies in each qPCR reaction was calculated according to the formula in [9]. Then the calibration curves were generated by the CFX Manager ${ }^{\mathrm{TM}}$ Software (Bio-Rad, USA) and the sensitivity of RT-qPCR was assessed.

\section{G. Specificity Test}

The specificity tests of RHDV2 RT-qPCR was performed through comparing the positive recombinant plasmids to RHDV(RHDV-Sch01), Pasteurella multocida, E.coli and Salmonella, EBHSV (pGM-T-EBHSV) etc common rabbit pathogens. RNA and DNA (cDNA) templates were prepared using a commonly reported method from [10].

\section{H. Repeatability Test}

To assess the repeatability of this qPCR, the intra-assay was performed though three recombinant plasmid dilutions (from $10^{-5}$ to $10^{-7}$ ) were detected in triplicate in the tests. The mean $\mathrm{Cq}$ values, standard deviation (SD) and percent coefficient of variation (\%CV) were calculated independently for each recombinant plasmid dilution in the tests.

\section{Primary Application Test}

5 RHDV experimentally samples infected with the RHDV-Sch01 and 25 clinical samples were detected by using this RT-qPCR method established above.

\section{RESULT}

\section{A. Construction of Recombinant Plasmids}

To develop the RT-qPCR for RHDV2 detection, a 435 bp-length target DNA fragment of RHDV2 VP60 gene was synthesized using over-lapping PCR(see Fig. 1,lane1), and the corresponding RHDV VP60 target fragment about 441 bp in the length was amplified by RT-PCR method (see Fig. 1,lane1).Then the two target fragments were cloned into pMD-19T Vector to construct recombinant plasmids named pMD-19T-RHDV2 and pMD-19T-RHDV separately, then the recombinant plasmids were identified using PCR ( same as Fig. 1)and sequencing as described above, The sequencing results showed the two VP60 target DNA fragment of RHDV2 and RHDV were both successfully synthesized and cloned into pMD-19T Vectors, which had a 100\% homology with the reference sequences(RHDV2 with KP129339 and RHDV with KF537692) published in GenBank. 


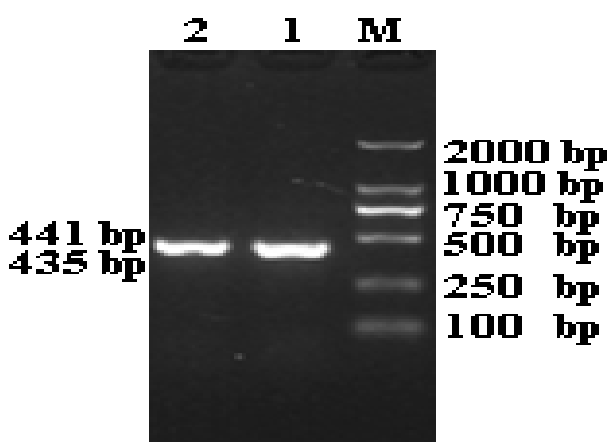

Figure 1. Amplification results of target DNA fragments M: DNA marker DL2000; 1: Overlapping-PCR products of RHDV2 VP60; 2: RT-PCR products of RHDV VP60;

\section{B. Optimization of RHDV2 RT-qPCR Reaction Conditions}

The RT-qPCR reaction conditions were determined based on the optimization of annealing degree. The results showed that the cycle thresholds (Cq Value) of positive controls in different degrees were all lower than 10(see Fig.2A, the left curves), only the Cq Values of negative controls1-3 were higher than 35 (see Fig. 2A, the right curves1-3), and all the amplification products had the similar melt curves with a $83^{\circ} \mathrm{C}$ Tm value(see Fig.2B). Then the optimal temperature and reaction time for the RT-qPCR were determined at $64.7{ }^{\circ} \mathrm{C}$ for $25 \mathrm{~s}$ for the specificity and amplification efficiency, and the $20 \mu \mathrm{L}$ reaction volume as described in the method section above was determined, the optimum RT reaction volume components and conditions were the same as the instructions of Primerscript RT reagent Kit.
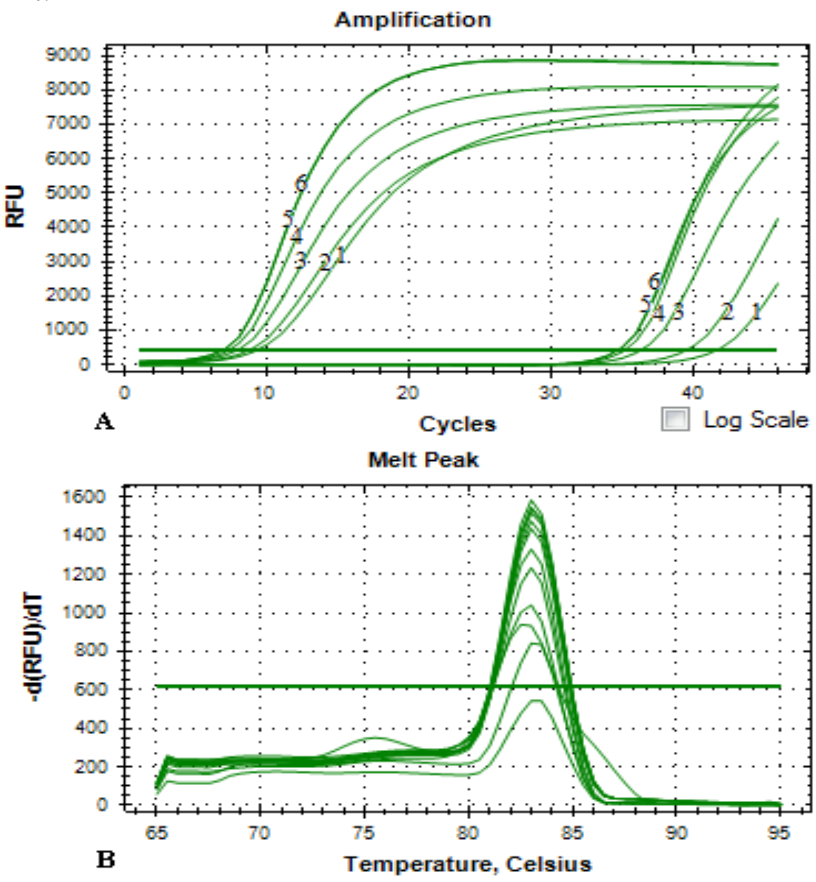

Figure 2. The test results of annealing degree Optimization.

A: The amplification curves of positive and negative control sanples; $\mathrm{B}$ :The melt curves of amplification prodcuts $1: 65^{\circ} \mathrm{C} ; 2: 64.7^{\circ} \mathrm{C} ; 3: 64{ }^{\circ} \mathrm{C}$; $4: 62.8^{\circ} \mathrm{C} ; 5: 60.2^{\circ} \mathrm{C} ; 6: 59^{\circ} \mathrm{C}$

\section{Establishment of Standard Curve}

The RT-qPCR was standardized with the pMD-19T-RHDV2(44.2ng $/ \mu \mathrm{L}, 1.28 \times 10^{10}$ copies $\left./ \mu \mathrm{L}\right)$ in a 10 -fold serial dilutions as above, then standard curve was generated by the CFX Manager ${ }^{\mathrm{TM}}$ Software from the Cq values obtained against the log of known copy numbers(diluted ranging from $10^{-2}$ to $10^{-9}$ ) (see Fig.3A and 3B), The slope value was -3.347 , the y-intercept value was 39.994(lower than the Cq of 50), E value was 99\%, and the $\mathrm{R}^{2}$ (correlation coefficient) reached $100 \%$. All the results indicated the performance of the RT-qPCR was linear ranging from 128 copies $/ \mu \mathrm{L}$ to $1.28 \times 10^{8}$ copies $/ \mu \mathrm{L}$ for pMD-19T-RHDV2.

\section{Sensitivity, Specificity and Repeatability of RHDV2 $R T-q P C R$}

The sensitivity, and specificity and repeatability of the RT-qPCR were assessed in the tests which were described in the methods section. The results showed the $\mathrm{Cq}$ Value of pMD-19T-RHDV2 diluted ranging from $10^{-9}$ to $10^{-10}(12.8$ copies/ $\mu \mathrm{L}$ and 1.28 copies $/ \mu \mathrm{L}$ ) were higher than 35 (see Fig.4, curve 5 Cq was 35.72), indicating that the detection limit of the qPCR was 128 copies/ $\mu \mathrm{L}$ of the target gene fragments. For the specificity, there was a expected amplification curve(see Fig.5, curve 1) that was only observed for the pMD-19T-RHDV2 detection, and no any amplification curves were obtained for the detection of RHDV(RHDV-Sch01), Pasteurella multocida, E.coli and Salmonella, EBHSV(pGM-T-EBHSV) etc common rabbit pathogens.(see Fig.5 curve2-6). Intra-assay variability was calculated by assessing the homogeneity among replicates of dilutions $10^{-5}$ to $10^{-7}$. The results showed the mean $\mathrm{Cq}$ values $(\mathrm{x} \pm \mathrm{SD})$ of the three dilutions were $23.37 \pm 0.062$, $26.59 \pm 0.088,29.48 \pm 0.140$, respectively. And their \%CV values $(0.27,0.33$ and 0.47$)$ disclosed a low variation (which were all lower than 0.8 ) indicating the high repeatability of the method. 

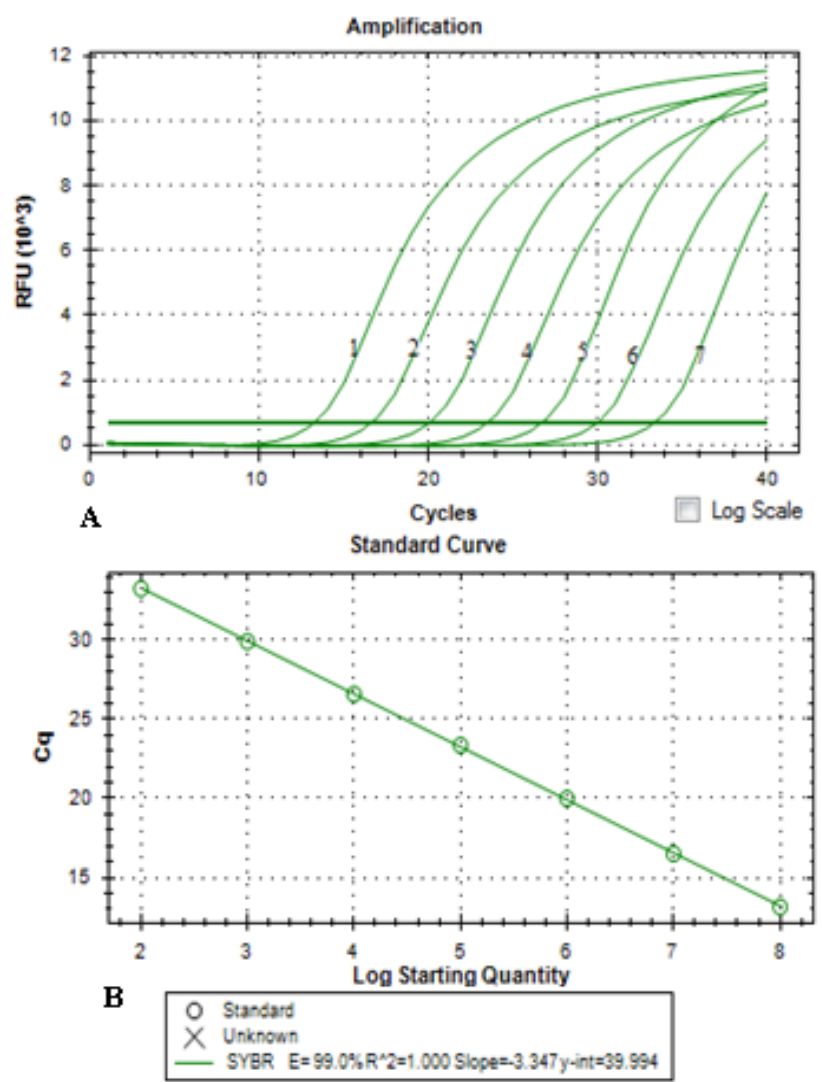

Figure 3. The establishment of standard curves of RHDV2 RT-qPCR A: The amplification curves of stadard sanples; B:The standard curves of RHDV2 RT-qPCR $1: 1.28 \times 10^{8}$ copies $/ \mu L ; 2: 1.28 \times 10^{7}$ copies $/ \mu \mathrm{L} ; 3$ : $1.28 \times 10^{6}$ copies $/ \mu \mathrm{L} ; 4: 1.28 \times 10^{5}$ copies $/ \mu \mathrm{L} ; 5: 1.28 \times 10^{4}$ copies $/ \mu \mathrm{L} ; 6$ : $1.28 \times 10^{3}$ copies $/ \mu \mathrm{L} ; 7: 1.28 \times 10^{2}$ copies $/ \mu \mathrm{L}$

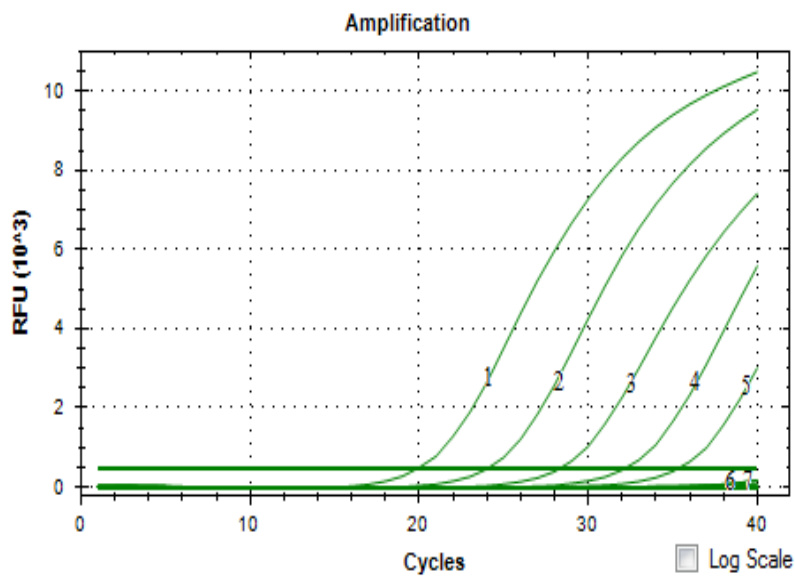

Figure 4. The sensitivity assay results of RHDV RT-qPCR 1:1.28 $\times 10^{5}$ copies $/ \mu \mathrm{L} ; 2: 1.28 \times 10^{4}$ copies $/ \mu \mathrm{L} ; 3: 1.28 \times 10^{3}$ copies $/ \mu \mathrm{L} ; 4$ : $1.28 \times 10^{2}$ copies $/ \mu \mathrm{L} ; 5: 1.28 \times 10^{1}$ copies $/ \mu \mathrm{L} ; 6: 1.28 \times 10^{0}$ copies $/ \mu \mathrm{L}$; 7:NTC(ddH2O)

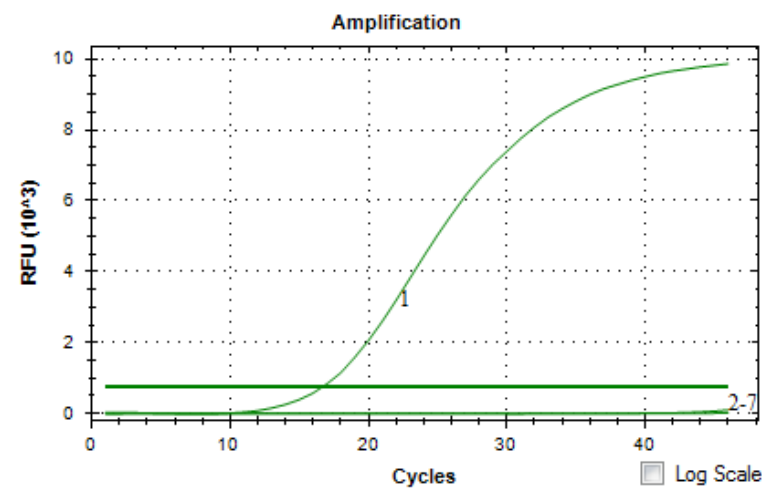

Figure 5. The specificity assay results of RHDV RT-qPCR 1: pMD-19T-RHDV2; 2: RHDV (RHDV-Sch01); 3: Pasteurella multocida; 4: E.coli; 5: Salmonella; 6: EBHSV (pGM-T-EBHSV); 7:NTC(ddH2O)

\section{E. Primary Application of RHDV2 RT-qPCR}

5 RHDV experimentally RHDV samples and 25 clinical samples were detected by this method, there were not any amplication for the 30 samples detection.

\section{Discussion AND CONCLUSIONS}

Rabbit hemorrhagic disease virus (RHDV), which belongs to genus Lagovirus of the family Caliciviridae, is a non-enveloped RNA virus with a diameter of about 36-40 $\mathrm{nm}$, and the genome consists of a positive-sense single-stranded RNA molecule of 7437nt in size. RHDV strains were divided into G1 - G6 6 different genotypes (group) according to the gene homology analysis[7]. RHDV strains still belongs to a kind of serotype, although there were exists certain antigenicity changes between different RHDV genotypes (especial RHDVa), because the traditional vaccine could be used for prevention. While RHDV2, also referred as RHDVb, was different with the 6 different genotypes RHDV (classical RHDV) in antigenicity and genotypes, and could affect younger rabbits, RHD-vaccinated rabbits and some hare species. And the molecular epidemiology of RHDV2 had revealed that it was rapidly replacing the previously circulating classical strains in France, Spain and Portugal[11,12].Therefore, RHDV2 not only brings economic loss to rabbit keeping, but also a serious threat to the ecosystem balance of the rabbit centered.

Considering the pathogen's characteristics (such as its high transmissibility, pathogenicity, and perniciousness) and there is no RHDV2 infection in China, 10 overlap PCR primers and 2 specific primers (see table 1 ) were designed and synthesized by collecting and analyzing a large number of RHDV2 molecular biological information. Then the conserved gene fragments about 435 bp of RHDV2VP60 were successfully synthesized by overlapping PCR. The process provides safe biomaterials for the further detection methods researches. At the same time, to prove that the reliability of the choice selecting the conserved gene fragments about 435 bp of RHDV2VP60 as template(cDNA), we also has cloned the RHDV VP60 target fragment which is corresponding to the conserved gene fragments about 435 bp of RHDV2VP60 by using 


\section{RT-PCR.}

In conclusion, the RT-qPCR assays described here exhibits good sensitivity, specificity and repeatability.The detection limit corresponding to 128 copies $/ \mu \mathrm{L}$ of objective gene segments, and there were no signs of amplification of common pathogens such as RHDV(RHDV-Sch01), Pasteurella multocida, E.coli and Salmonella, EBHSV(pGM-T-EBHSV) etc. The RT-qPCR performance was linear ranging from 128 copies $/ \mu \mathrm{L}$ to $1.28 \times 10^{8} \mathrm{copies} / \mu \mathrm{L}$ for the target gene segments, and with a low variation of \%CV values. In all, the study is the first report about the SYBR I GreenRT-qPCR assay for RHDV2 detection, and supplies a useful technology to keep in reserve to help prevent RHDV2 spread in China and for its control.

\section{ACKNOWLEDGEMENT}

This study was supported by Grants from the National Natural Science Foundation of China (31402222), the planning subject of 'the twelfth five-year-plan' in national science and technology for the rural development in China(2013BAD12B04), and Sichuan province science and technology support plan (2016NZ0002).Liu Yadong, Wang Bo, Li Yan and Meng Zhengqun should also be considered as first authors, Corresponding author: Wang Yin, Yang Zexiao.

\section{REFERENCES}

[1] G. Le Gall, C.Arnauld, E.Boilletot, J.P.Morisse and D.Rasschaert, "Molecular epidemiology of rabbit haemorrhagic disease virus outbreaks in France during 1988 to 1995,” J Gen Virol, vol. 79, Jan 1998, pp. 11-16, doi: 10.1099/0022-1317-79-1-11.

[2] B Gromadzka, B Szewczyk, G Konopa, A Fitzner and A Kesy, "Recombinant VP60 in the form of virion-like particles as a potential vaccine against rabbit hemorrhagic disease virus," Acta Biochim Pol. vol. 53, May 2006, pp. 371-376.

[3] F Chen. Veterinary Lemology, the 5th ed Beijing:China Agriculture Press,Apr.2008,pp.410-412.

[4] M.T. McIntosh, S.C. Behan, F.M. Mohamed, Z.Lu, K.E. Moran, T. G.
Burrage, J.G. Neilan, G.B. Ward, G. Botti, L.Capucci and S.A Metwally, "A pandemic strain of calicivirus threatens rabbit industries in the Americas,” Virology Journal, vol. 4, Oct 2007, pp.96-108, doi:10.1186/1743-422X-4-96

[5] L Yang, F Wang, B Hu, J Xue, Y Hu, B Zhou, D Wang and W Xu "Development of an RT-PCR for rabbit hemorrhagic disease virus (RHDV) and the epidemiology of RHDV in three eastern provinces of China,” J Virol Methods,vol. 151, Jul 2008 ,pp.24-29, doi:10.1016/j.jviromet.2008.04.003

[6] G Le Gall-Reculé, A Lavazza, S Marchandeau, S Bertagnoli, F Zwingelstein, P Cavadini,N Martinelli, G Lombardi, J LGuérin,E Lemaitre,A Decors, S Boucher, B Le Normand and L Capucci, "Emergence of a new lagovirus related to Rabbit Haemorrhagic Disease Virus,” Veterinary Research, vol. 44, Sep 2013,pp.81, doi:10.1186/1297-9716-44-81

[7] J Bárcena, B Guerra, I Angulo, J González, F Valcárcel, C.P. Mata, J.R. Castón, E .Blanco and A .Alejo, "Comparative analysis of rabbit hemorrhagic disease virus (RHDV) and new RHDV2 virus antigenicity, using specific virus-like particles,” Vet Res, vol. 46, Sep 2015,pp.106. doi: 10.1186/s13567-015-0245-5.

[8] A Martin-Alonso, N Martin-Carrillo, K Garcia-Livia, B Valladares and P Foronda, "Emerging rabbit haemorrhagic disease virus 2 (RHDV2) at the gates of the African continent," Infect Genet Evol, vol. 44,Oct 2016,pp.46-50. doi: 10.1016/j.meegid.2016.06.034.

[9] Z Yang, B Wang, Q Xu, X Yao, Zhu L, Z. Xu, K. Wang, G. Li, S. Peng and Y.Wang, "Designment and Evaluation of the Primers for Rift Valley Fever (RVF) Virus RT- PCR Detection,” Advanced Materials Research, vol. 989-994,Dec 2014,pp.1115-1119, doi: 10.4028/www.scientific.net/AMR.989-994.1115

[10] Z. Yang, G.Li, Y. Hou, X. Yao, R. Ren, H. Ya, S. Peng, X. Lin and Y. Wang, "Artificial Synthesis of Conserved Segment S Gene Fragment of Rift Valley Fever Virus and Preliminary Study of Its Reverse Transcription Loop-Mediated Isothermal Amplification Detection Method,” Tropical Journal of Pharmaceutical Research, vol. 14,Dec 2015,pp. 2193-2200.

[11] K.P. Dalton, I.Nicieza, J.Abrantes, P.J.Esteves and F.Parra, "Spread of new variant RHDV in domestic rabbits on the Iberian Peninsula," Veterinary Microbiology, vol. 169, Feb 2014, pp.67-73, doi: 10.1016/j.vetmic.2013.12.015.

[12] AM Lopes,J Correia, J Abrantes, P Melo,M Ramada, MJ Magalhães,PC Alves and PJ Esteves, "Is the new variant RHDV replacing genogroup 1 in Portuguese wild rabbit populations?” Viruses, vol. 7, Dec 2014, pp.27-36, doi: 10.3390/v7010027. 